\title{
An analytic approach to the spectral characterization of the radical
}

\author{
by \\ Graham R. Allan (Cambridge)
}

\begin{abstract}
We give a simple complex-variable proof of an old result of Zemánek and Le Page on the radical of a Banach algebra. Incidentally, the argument also proves a recent result of Harris and Kadison.
\end{abstract}

Throughout this paper, $A$ is a complex unital Banach algebra, $J \equiv J(A)$ is the Jacobson radical of $A$ and $N \equiv N(A)$ is the set of quasi-nilpotent elements of $A$, i.e. $N=\{x \in A: \operatorname{Sp} x=\{0\}\}$. For elements $a, b$ of an algebra, write $[a, b]=a b-b a$, the commutator of $a$ and $b$.

In Zemánek's proof of his well known characterization of the radical ([5], [6], [7]), an important step was to prove the following theorem.

Theorem 1. Let $x \in A$ have the property that $[x, a] \in N$ for every $a \in A$. Then $[x, a] \in J$ for every $a \in A$.

This result had been independently proved by Le Page [3] by essentially the same method, using the Jacobson density theorem.

In a recent paper [2], Harris and Kadison define the notion of a Schurian algebra. As well as proving a number of properties of Schurian algebras, they give examples, in particular they prove that every complex Banach algebra with identity is Schurian. We do not need, here, to define "Schurian", but instead give the result explicitly as follows:

Theorem 2 ([2]). Let $A$ be a complex Banach algebra with 1, let $L$ be a maximal left ideal of $A$ and let $x$ be an element of $A$ such that $L x \subseteq L$. Then there is some $\lambda \in \mathbb{C}$ such that $x-\lambda 1 \in L$.

REMARK. The converse to Theorem 2 is, of course, trivial; it is also clear that, for given $L$ and $x$, the complex number $\lambda$ is uniquely determined.

While the proof in [2] is quite straightforward, it seems interesting to give a proof that uses just elementary complex-variable theory. In fact in

2000 Mathematics Subject Classification: Primary 46H10.

Key words and phrases: Banach algebra, radical. 
[1, Theorem 2.3] we proved the special case of Theorem 2 in which $x$ is supposed to belong to the centre of $A$. Our present proof of Theorem 2 is a simple adaptation of that earlier proof.

In this paper we shall first give the simple proof of Theorem 2 and then use that result, with a few simple algebraic lemmas, to give a proof of Theorem 1 without the use of representation theory.

Proof of Theorem 2. First a remark: suppose that $x \notin L$ but that $y x \in L$ for some $y \in A$. Then we claim that $y \in L$, for otherwise there is some $z \in A$ with $z y-1 \in L$ and then, since $L x \subseteq L$, it follows that $z y x-x \in L$. But then we would have $x \in L$, contrary to hypothesis.

To prove the main result: suppose that, for every $\lambda \in \mathbb{C}$ we have $x-\lambda 1$ $\notin L$. Then, for every $\lambda \in \mathbb{C}$ there is some $y(\lambda) \in A$ with $y(\lambda)(x-\lambda 1)-1 \in L$. Since, for every $\lambda \in \mathbb{C}, L(x-\lambda 1) \subseteq L$ it follows from the first paragraph of the proof that $y(\lambda)$ is uniquely determined modulo $L$.

More explicitly, let $\pi: A \rightarrow A / L$ be the quotient map and define

$$
f(\lambda)=\pi(y(\lambda)) \quad(\lambda \in \mathbb{C}),
$$

where $y(\lambda)$ is any element of $A$ such that $y(\lambda)(x-\lambda 1)-1 \in L$. Then $f$ is a well-defined $A / L$-valued function on $\mathbb{C}$.

Now let $\mu \in \mathbb{C}$ and choose $y \in A$ so that $y(x-\mu 1)-1 \in L$. For $|\lambda-\mu|<\|y\|^{-1}$, the element $1+(\mu-\lambda) y$ is invertible. A simple computation shows that $(1+(\mu-\lambda) y)^{-1} y(x-\lambda 1)-1 \in L$; i.e. for $|\lambda-\mu|<\|y\|^{-1}$, we may take $y(\lambda)=(1+(\mu-\lambda) y)^{-1} y$, which is holomorphic in $\lambda$. It follows that $f$ is a holomorphic $A / L$-valued function on $\mathbb{C}$. For $|\lambda|>\|x\|$ we may take $y(\lambda)=(x-\lambda 1)^{-1}$; it being well known that $(x-\lambda 1)^{-1} \rightarrow 0$ as $|\lambda| \rightarrow \infty$, it follows that also $f(\lambda) \rightarrow 0$ as $|\lambda| \rightarrow \infty$.

It then follows from Liouville's theorem that $f(\lambda) \equiv 0$, so that each $y(\lambda)$ is in $L$ and then $1 \in L$ and $L=A$, which is a contradiction. This completes the proof.

Lemma 3. Let $L$ be a maximal left ideal of $A$ and let $x \in A$. Suppose that $[l, x] \in N$ for every $l \in L$; then $L x \subseteq L$.

Proof. Suppose that $[l, x] \in N$ for every $l \in L$, but that $L x \nsubseteq L$. There is thus some $m \in L$ with $m x \notin L$ and then, by maximality of $L$, there is some $b \in A$ such that $b m x-1 \in L$. Hence $[b m, x]-1=(b m x-1)-x b m \in L$; but, since $b m \in L$, the hypothesis implies that $[b m, x] \in N$, so that $[b m, x]-1$ is invertible, contrary to $L$ being a proper left ideal.

Corollary 4. Let $x \in A$ have the property that $[x, a] \in N$ for every $a \in A$ and let $L$ be a maximal left ideal of $A$. Then there is a unique $\lambda \in \mathbb{C}$ such that $x-\lambda 1 \in L$. 
Proof. It is clear that there is at most one $\lambda \in \mathbb{C}$ for which $x-\lambda 1 \in L$. By Lemma 3 we have $L x \subseteq L$ and the result is now immediate from Theorem 2 .

In what follows $G \equiv G(A)$ is the group of invertible elements of the Banach algebra $A$. The next lemma is elementary algebra.

Lemma 5. Let $L$ be a maximal left ideal of $A$ and let $x \in A$. Suppose that for every $u \in G(A)$ there is some $\lambda=\lambda(u) \in \mathbb{C}$ with $(x-\lambda 1) u \in L$. Then there is a unique $\lambda \in \mathbb{C}$ with $(x-\lambda 1) A \subseteq L$.

Proof. Note that, for each $u \in G$, the $\lambda=\lambda(u) \in \mathbb{C}$ with $(x-\lambda 1) u \in L$ is unique, since $u \notin L$. By hypothesis, there is a unique $\mu \in \mathbb{C}$ with $x-\mu 1 \in L$; we shall show that $\lambda(u)=\mu$ for every $u \in G$. Thus, let $u \in G$.

CASE (i): there are $\alpha, \beta \in \mathbb{C}$, not both zero, with $\alpha 1+\beta \mu \in L$. Necessarily $\alpha \beta \neq 0$ and it follows that $(x-\mu 1) u \in L$, i.e. that $\lambda(u)=\mu$.

CASE (ii): the set $\{1, u\}$ is linearly independent modulo L. Choose $\alpha \in$ $\mathbb{C} \backslash\{0\}$ with $u+\alpha 1 \in G$ (e.g. any $\alpha$ with $|\alpha|>\|u\|$ ). Then there are $\gamma, \delta \in \mathbb{C}$ with $(x-\gamma 1) u \in L,(x-\delta 1)(u+\alpha 1) \in L$ and we already have $x-\mu 1 \in L$. A simple calculation then shows that $(\gamma-\delta) u+\alpha(\mu-\delta) 1 \in L$ so that, by the hypothesis of Case (ii), $\gamma=\delta=\mu$.

Thus $(x-\mu 1) G \subseteq L$ and so also, since $A=G+G,(x-\mu 1) A \subseteq L$.

Lemma 6. Let $x \in A$ have the property that $[x, a] \in N$ for every $a \in A$ and let $L$ be a maximal left ideal of $A$. Then there is a unique $\lambda \in \mathbb{C}$ with $(x-\lambda 1) A \subseteq L$.

Proof. By Lemma 5, it is sufficient to show that for each $u \in G$ there is some $\lambda \in \mathbb{C}$ with $(x-\lambda 1) u \in L$.

But, for any $u \in G,(x-\lambda 1) u \in L$ if and only if $u^{-1}(x-\lambda 1) u \in L$, i.e. $u^{-1} x u-\lambda 1 \in L$. Also, for every $a \in A,\left[u^{-1} x u, a\right]=u^{-1}\left[x, u a u^{-1}\right] u \in N$. So the result follows from Corollary 4.

Proof of Theorem 1. Let $P$ be a primitive ideal of $A$, so that there is a maximal left ideal $L$ with

$$
P=L: A \equiv\{a \in A: a A \subseteq L\} .
$$

By Lemma 6 , there is $\lambda \in \mathbb{C}$ with $x-\lambda 1 \in P$. Thus $[x, a]=[x-\lambda 1, a] \in P$ for every $a \in A$. Since $J(A)$ is the intersection of all the primitive ideals of $A$, the result is proved.

Corollary 7 ([4, Lemma 1], [5, Lemma 1.22]). Let $x \in A$; then $x \in$ $J(A)$ if and only if both $x \in N(A)$ and $[x, a] \in N(A)$ for all $a \in A$.

Proof. For the non-trivial implication, suppose that $[x, a] \in N(A)(a \in A)$. Since $x \in N(A)$ then $x+J \in N(A / J)$; but also by Theorem $1, x+J$ is in the centre of $A / J$. It follows that $x+J \in J(A / J)=\{0\}$, i.e. $x \in J$. 


\section{References}

[1] G. R. Allan, Ideals of vector-valued functions, Proc. London Math. Soc. (3) 18 (1968), 193-216.

[2] L. A. Harris and R. V. Kadison, Schurian algebras and spectral additivity, J. Algebra 180 (1996), 175-186.

[3] C. Le Page, Sur quelques conditions entraînant la commutativité dans les algèbres de Banach, C. R. Acad. Sci. Paris Sér. A 265 (1967), 235-237.

[4] Z. Słodkowski, W. Wojtyński and J. Zemánek, A note on quasinilpotent elements of a Banach algebra, Bull. Acad. Polon. Sci. Sér. Sci. Math. Astronom. Phys. 25 (1977), 131-134.

[5] J. Zemánek, Properties of the spectral radius in Banach algebras, $\mathrm{PhD}$ thesis, Inst. Math., Polish Acad. Sci., Warszawa, 1977.

[6] - A note on the radical of a Banach algebra, Manuscripta Math. 20 (1977), 191-196.

[7] - Spectral radius characterizations of commutativity in Banach algebras, Studia Math. 61 (1977), 257-268.

Department of Pure Mathematics and Mathematical Statistics

Centre for Mathematical Sciences

Wilberforce Road

Cambridge CB3 0WB, U.K.

E-mail: G.R.Allan@dpmms.cam.ac.uk

Received 25 May 2004

Revised version 26 October 2004

Editorial note. Further results related and analogous to Theorem 1 have been subsequently obtained in [A1], [A2], [BM], [B], [G], [KS] and [TSh]. It would be interesting to see if the author's method can be applied or modified in these related cases, and even to find a unified approach to all of them.

[A1] B. Aupetit, A Primer on Spectral Theory, Springer, 1991.

[A2] - Spectral characterization of the radical in Banach and Jordan-Banach algebras, Math. Proc. Cambridge Philos. Soc. 114 (1993), 31-35.

[BM] M. Brešar and M. Mathieu, Derivations mapping into the radical, III, J. Funct. Anal. 133 (1995), 21-29.

[B] R. M. Brits, On the multiplicative spectral characterization of the Jacobson radical, Quaestiones Math., to appear.

[G] S. Grabiner, The spectral diameter in Banach algebras, Proc. Amer. Math. Soc. 91 (1984), 59-63.

[KS] A. Katavolos and C. Stamatopoulos, Commutators of quasinilpotents and invariant subspaces, Studia Math. 128 (1998), 159-169.

[TSh] Yu. V. Turovskiı̌ and V. S. Shul'man, Conditions for the massiveness of the range of a derivation of a Banach algebra and of associated differential operators, Mat. Zametki 42 (1987), 305-314 (in Russian); English transl.: Math. Notes 42 (1987), $669-674$. 